\title{
Uma analise da utilização de serviços de saúde por sistema de financiamento: Brasil 1998 -2008
}

\author{
An analysis of use of the health services by financing system: \\ Brazil 1998-2008
}

Silvia Marta Porto ${ }^{1}$

Maria Alicia Dominguez Ugá ${ }^{1}$

Rodrigo da Silva Moreira ${ }^{2}$

${ }^{1}$ Escola Nacional de Saúde Pública, Fiocruz. Rua Leopoldo Bulhões $1.4807^{\circ}$ andar, Manguinhos. 22241-210 Rio de Janeiro RJ.sporto@ensp.fiocruz.br ${ }^{2}$ Instituto de Comunicação e Informação Científica e Tecnológica em Saúde, Fiocruz.
Abstract This article analyzes the use of health services from the perspective of financing based on PNAD/IBGE micro-data related to 1998, 2003 and 2008. Among the main results, the following can be highlighted: 1) The Unified Health System (SUS) continues to be the major financing agent of most consultations and hospitalizations in Brazil; its participation increased significantly between 1998 and 2003 and remained almost stable between 2003 and 2008; 2) SUS participation in financing the use of the health services has been predominant in all Brazilian regions, especially in the North and North-East, which feature the most precarious socio-economic and health conditions; 3) SUS is the major financing agent of the two extreme levels of complexity of health care: primary care and high complexity services. 4) In spite of a significant rise in utilization rates of SUS services for consultations and hospitalizations, great inequities can still be observed between the population that exclusively uses SUS and that which has private health insurance; 5) There has been an increase in the use of SUS health services by part of the population with private health insurance plans.

Key words Financing, The use of health services
Resumo Este artigo analisa, a partir de microdados de 1998, 2003 e 2008 da PNAD/IBGE, a utilização de serviços de saúde sob a perspectiva de seu financiamento Entre os principais resultados da análise, destacam-se os seguintes: 1) o SUS continua financiando a maioria dos atendimentos $e$ das internações realizados no País, participação que aumentou significativamente entre $1998 \mathrm{e}$ 2003, mantendo-se praticamente estável entre 2003 e 2008; 2) a participação do SUS no financiamento da utilização dos serviços de saúde foi preponderante em todas as regiões, sendo maior nas regiões Norte e Nordeste com situações sanitárias e sócio-econômicas mais precárias; 3) o SUS é o principal financiador dos dois níveis extremos de complexidade da atenção à saúde: o de atenção básica e o da alta complexidade; 4) apesar do aumento verificado nas taxas de utilização do SUS, para atendimentos e internações, ainda persistem grandes inequidades no uso de serviços de saúde entre a população atendida pelo SUS e a população beneficiaria de planos e seguros de saúde privados; 5) foi observado um aumento na utilização de serviços do SUS por parte dessa população com asseguramento privado.

Palavras-chave Financiamento, Utilização de serviços de saúde 


\section{Introdução}

Este artigo tem por objetivo analisar, a partir de dados das PNAD (Pesquisa Nacional por Amostra de Domicílio) 1998, 2003 e 2008, a utilização de serviços de saúde segundo seu sistema de financiamento: se pelo Sistema Único de Saúde (SUS), ou seja, pelo sistema de proteção vinculado ao sistema nacional de saúde, se por planos e seguros privados de saúde (asseguramento privado) ou se mediante a compra de serviços, com pagamento direto.

É analisado o número de eventos de utilização de serviços cobertos por cada segmento; portanto, os resultados nada indicam em relação ao volume de recursos movimentados por cada sistema de financiamento.

Aos três segmentos correspondem três formas de financiamento: o SUS é financiado por recursos das três esferas de governo; os planos e seguros de saúde privados são financiados pelos pagamentos efetuados pelos beneficiários individuais e, no caso dos beneficiários de planos coletivos, pelos pagamentos efetuados por eles e/ ou pelas empresas que os empregam. Finalmente, no terceiro segmento se encontram aqueles que utilizam serviços de saúde mediante o pagamento direto no ato do consumo, conhecido na literatura internacional como out-of-pocket.

Cabe destacar que os resultados obtidos mostram a importância da PNAD para acompanhar e mensurar, ao mesmo tempo, a evolução do SUS em termos de sua participação no financiamento da utilização dos serviços de saúde, bem como o correspondente comportamento do segmento de saúde suplementar e do gasto privado.

\section{Metodologia}

Para analisar os dados da PNAD utilizou-se das variáveis de seu desenho amostral (peso, estratificação e conglomeração), procedimento descrito em Silva et al. ${ }^{1}$.

As informações da pesquisa foram trabalhadas seguindo a mesma metodologia utilizada em Porto et al. ${ }^{2}$. Conforme apontado no referido artigo, o questionário contempla as seguintes perguntas, que permitem identificar o sistema de financiamento pelo qual se deu a utilização de serviços:

1) este atendimento de saúde foi coberto por algum plano de saúde? [alternativas: Sim ou Não];

2) pagou algum valor por este atendimento de saúde [...]? [alternativas: Sim ou Não];
3) este atendimento de saúde foi feito pelo SUS? [alternativas: Sim, Não ou Não sabe).

As mesmas perguntas e alternativas de resposta foram elaboradas em relação às internações hospitalares. Deve-se destacar que as perguntas não foram excludentes e que, portanto, um mesmo indivíduo pode ter respondido Sim a mais de uma.

A opção metodológica foi a de considerar:

a) atendimentos ou internações cobertos pelo SUS: todas as respostas Sim à pergunta 3 , independentemente de ter respondido positivamente à pergunta 2 , e que tenha respondido Não à pergunta 1 ;

b) atendimentos ou internações cobertos por planos ou seguros de saúde: aqueles que disseram Sim à pergunta 1 , independentemente de ter respondido Sim à pergunta 2 , e Não ou Não sabe à pergunta 3 ;

c) atendimentos ou internações financiados diretamente com recursos do paciente foram considerados aqueles que responderam Sim à pergunta 2, excluindo aqueles que responderam Sim às perguntas $1 \mathrm{e} / \mathrm{ou} 3$.

Da mesma forma que no artigo mencionado, para trabalhar as informações sobre a utilização dos serviços de saúde com as da renda, utilizouse o rendimento mensal familiar sem agregados que, conforme o glossário da publicação do $\mathrm{IBGE}^{3}$, é a soma dos rendimentos mensais dos componentes da família, exclusive os das pessoas com menos de 10 anos de idade e os daquelas cuja condição na família é de pensionista, empregado doméstico ou parente do empregado doméstico.

Em decorrência dos erros associados as estimativas de uma pesquisa amostral, adotou-se no artigo a utilização do coeficiente de variação (CV) para se identificar as estimativas de maior imprecisão. Estas estimativas foram classificadas em dois grupos: pouco precisas (CV de 0,25 a 0,5 ) e imprecisas (CV acima de 0,5).

\section{Resultados}

\section{Atendimentos}

\section{Atendimentos por tipos selecionados de atendimento, segundo sistema de financiamento}

Segundo a PNAD 2008, o SUS financiou nesse período os atendimentos de $58,6 \%$ das pessoas que os utilizaram no país. Seu maior protagonismo se deu nos atendimentos por quimio e radioterapia, hemodiálise e hemoterapia (finan- 
ciando 73,3\% dos mesmos), nas consultas médicas $(62,7 \%)$ e nos exames complementares $(59,2 \%)$. Portanto, o SUS financiou não só atendimentos de mais baixo custo, mas também -e principalmente- os mais onerosos (Tabela 1).

Vale notar que, segundo Viacava ${ }^{4}, 43 \%$ das mamografias realizadas no país foram financiadas pelo SUS, dando-se a maior participação do setor público nas regiões mais carentes (49\% no Nordeste e $47 \%$ no Norte). Ainda segundo o mesmo autor, verifica-se um protagonismo ain- da mais importante (53\%) do SUS no que diz respeito ao financiamento dos exames Papanicolau, o qual deve explicar, pelo menos em parte, os dados acima apontados.

A participação dos planos de saúde no financiamento dos atendimentos é pouco expressiva, representando apenas $27 \%$ dos casos. Vale destacar a baixa participação $(24,2 \%)$ deste segmento de saúde suplementar no financiamento dos casos tratados por quimio, radioterapia, hemodiálise e hemoterapia.

Tabela 1. Atendimentos por tipos selecionados de atendimento, região e decil de renda, segundo financiamento - Brasil, 1998-2003-2008.

\begin{tabular}{|c|c|c|c|c|c|c|}
\hline & Ano & SUS & $\begin{array}{c}\text { Plano } \\
\text { Privados }\end{array}$ & $\begin{array}{c}\text { Gasto Privado } \\
\text { Direto } \\
\end{array}$ & Total & N Total \\
\hline \multicolumn{7}{|l|}{ Tipo de atendimento } \\
\hline \multirow[t]{3}{*}{ Consulta médica } & 1998 & $58,6 \%$ & $32,9 \%$ & $8,5 \%$ & $100 \%$ & 12.184 .354 \\
\hline & 2003 & $63,6 \%$ & $29,5 \%$ & $6,9 \%$ & $100 \%$ & 17.074 .681 \\
\hline & 2008 & $62,8 \%$ & $29,6 \%$ & $7,7 \%$ & $100 \%$ & 18.247 .855 \\
\hline \multirow[t]{3}{*}{ Consulta odontológica } & 1998 & $28,7 \%$ & $19,8 \%$ & $51,5 \%$ & $100 \%$ & 1.866 .165 \\
\hline & 2003 & $32,6 \%$ & $18,6 \%$ & $48,9 \%$ & $100 \%$ & 1.920 .440 \\
\hline & 2008 & $30,5 \%$ & $17,9 \%$ & $51,6 \%$ & $100 \%$ & 3.649 .259 \\
\hline \multirow{3}{*}{$\begin{array}{l}\text { Quimioterapia, radioterapia, } \\
\text { hemodiálise, ou hemoterapia }\end{array}$} & 1998 & N/A & N/A & N/A & N/A & N/A \\
\hline & 2003 & $75,0 \%$ & $19,4 \%$ & $5,7 \% *$ & $100 \%$ & 94.911 \\
\hline & 2008 & $73,3 \%$ & $24,2 \%$ & $2,5 \%$ ** & $100 \%$ & 91.290 \\
\hline \multirow[t]{3}{*}{ Cirurgia em ambulatório } & 1998 & $51,7 \%$ & $40,6 \%$ & $7,8 \%$ & $100 \%$ & 173.136 \\
\hline & 2003 & $58,1 \%$ & $32,8 \%$ & $9,2 \%$ & $100 \%$ & 196.629 \\
\hline & 2008 & $51,7 \%$ & $36,6 \%$ & $11,7 \%$ & $100 \%$ & 212.281 \\
\hline \multirow[t]{3}{*}{ Exames complementares } & 1998 & $51,5 \%$ & $39,7 \%$ & $8,9 \%$ & $100 \%$ & 1.153 .746 \\
\hline & 2003 & $58,7 \%$ & $33,4 \%$ & $7,9 \%$ & $100 \%$ & 1.422 .221 \\
\hline & 2008 & $59,2 \%$ & $30,2 \%$ & $10,6 \%$ & $100 \%$ & 1.015 .953 \\
\hline \multirow[t]{3}{*}{ Total 1} & 1998 & $56,1 \%$ & $30,4 \%$ & $13,5 \%$ & $100 \%$ & 17.514 .524 \\
\hline & 2003 & $61,4 \%$ & $27,7 \%$ & $10,9 \%$ & $100 \%$ & 23.234.227 \\
\hline & 2008 & $58,6 \%$ & $27,0 \%$ & $14,4 \%$ & $100 \%$ & 25.513 .009 \\
\hline \multicolumn{7}{|l|}{ Região } \\
\hline \multirow[t]{3}{*}{ Norte } & 1998 & $67,9 \%$ & $17,8 \%$ & $14,3 \%$ & $100 \%$ & 814.609 \\
\hline & 2003 & $74,1 \%$ & $16,3 \%$ & $9,7 \%$ & $100 \%$ & 1.135 .918 \\
\hline & 2008 & $71,4 \%$ & $15,7 \%$ & $13,0 \%$ & $100 \%$ & 1.515 .920 \\
\hline \multirow[t]{3}{*}{ Nordeste } & 1998 & $71,3 \%$ & $19,2 \%$ & $9,5 \%$ & $100 \%$ & 4.257 .844 \\
\hline & 2003 & $74,9 \%$ & $16,0 \%$ & $9,0 \%$ & $100 \%$ & 5.798 .868 \\
\hline & 2008 & $71,3 \%$ & $16,3 \%$ & $12,4 \%$ & $100 \%$ & 6.177 .710 \\
\hline \multirow[t]{3}{*}{ Sudeste } & 1998 & $47,8 \%$ & $39,4 \%$ & $12,7 \%$ & $100 \%$ & 8.153 .075 \\
\hline & 2003 & $54,9 \%$ & $35,4 \%$ & $9,7 \%$ & $100 \%$ & 10.863 .323 \\
\hline & 2008 & $51,6 \%$ & $34,3 \%$ & $14,1 \%$ & $100 \%$ & 11.938 .724 \\
\hline \multirow[t]{3}{*}{ Sul } & 1998 & $54,4 \%$ & $27,3 \%$ & $18,3 \%$ & $100 \%$ & 3.058 .607 \\
\hline & 2003 & $56,4 \%$ & $28,2 \%$ & $15,4 \%$ & $100 \%$ & 3.902 .480 \\
\hline & 2008 & $55,2 \%$ & $26,7 \%$ & $18,2 \%$ & $100 \%$ & 4.159 .877 \\
\hline \multirow[t]{3}{*}{ Centro-Oeste } & 1998 & $54,8 \%$ & $25,2 \%$ & $20,0 \%$ & $100 \%$ & 1.230 .389 \\
\hline & 2003 & $60,0 \%$ & $24,7 \%$ & $15,3 \%$ & $100 \%$ & 1.533 .638 \\
\hline & 2008 & $58,6 \%$ & $25,0 \%$ & $16,5 \%$ & $100 \%$ & 1.720 .778 \\
\hline \multirow[t]{3}{*}{ Total } & 1998 & $56,1 \%$ & $30,4 \%$ & $13,5 \%$ & $100 \%$ & 17.514 .524 \\
\hline & 2003 & $61,4 \%$ & $27,7 \%$ & $10,9 \%$ & $100 \%$ & 23.234 .227 \\
\hline & 2008 & $58,6 \%$ & $27,0 \%$ & $14,4 \%$ & $100 \%$ & 25.513 .009 \\
\hline
\end{tabular}




\begin{tabular}{|c|c|c|c|c|c|c|}
\hline & Ano & SUS & $\begin{array}{c}\text { Plano } \\
\text { Privados }\end{array}$ & $\begin{array}{c}\text { Gasto Privado } \\
\text { Direto }\end{array}$ & Total & N Total \\
\hline \multicolumn{7}{|c|}{ Decil de renda } \\
\hline \multirow[t]{3}{*}{1} & 1998 & $88,5 \%$ & $5,6 \%$ & $5,8 \%$ & $100 \%$ & 1.989 .968 \\
\hline & 2003 & $92,5 \%$ & $3,6 \%$ & $3,9 \%$ & $100 \%$ & 2.072 .932 \\
\hline & 2008 & $87,6 \%$ & $5,5 \%$ & $6,9 \%$ & $100 \%$ & 3.132 .573 \\
\hline \multirow[t]{3}{*}{2} & 1998 & $89,4 \%$ & $4,3 \%$ & $6,3 \%$ & $100 \%$ & 1.097 .414 \\
\hline & 2003 & $90,2 \%$ & $4,3 \%$ & $5,5 \%$ & $100 \%$ & 2.519 .768 \\
\hline & 2008 & $86,4 \%$ & $5,9 \%$ & $7,7 \%$ & $100 \%$ & 1.553 .149 \\
\hline \multirow[t]{3}{*}{3} & 1998 & $82,9 \%$ & $7,9 \%$ & $9,2 \%$ & $100 \%$ & 1.613 .301 \\
\hline & 2003 & $85,8 \%$ & $7,4 \%$ & $6,9 \%$ & $100 \%$ & 1.841 .735 \\
\hline & 2008 & $83,0 \%$ & $8,4 \%$ & $8,6 \%$ & $100 \%$ & 2.247 .721 \\
\hline \multirow[t]{3}{*}{4} & 1998 & $77,5 \%$ & $12,0 \%$ & $10,5 \%$ & $100 \%$ & 1.541 .038 \\
\hline & 2003 & $81,9 \%$ & $9,6 \%$ & $8,4 \%$ & $100 \%$ & 2.207 .835 \\
\hline & 2008 & $75,9 \%$ & $12,1 \%$ & $12,0 \%$ & $100 \%$ & 2.501 .825 \\
\hline \multirow[t]{3}{*}{5} & 1998 & $68,9 \%$ & $19,2 \%$ & $11,8 \%$ & $100 \%$ & 1.778 .307 \\
\hline & 2003 & $74,5 \%$ & $16,2 \%$ & $9,3 \%$ & $100 \%$ & 2.410 .606 \\
\hline & 2008 & $70,4 \%$ & $16,4 \%$ & $13,3 \%$ & $100 \%$ & 2.417 .044 \\
\hline \multirow[t]{3}{*}{6} & 1998 & $61,9 \%$ & $26,1 \%$ & $12,1 \%$ & $100 \%$ & 1.594 .537 \\
\hline & 2003 & $68,5 \%$ & $20,7 \%$ & $10,8 \%$ & $100 \%$ & 2.057 .755 \\
\hline & 2008 & $63,7 \%$ & $21,6 \%$ & $14,7 \%$ & $100 \%$ & 2.688 .415 \\
\hline \multirow[t]{3}{*}{7} & 1998 & $52,2 \%$ & $34,5 \%$ & $13,2 \%$ & $100 \%$ & 1.718 .482 \\
\hline & 2003 & $57,9 \%$ & $29,7 \%$ & $12,4 \%$ & $100 \%$ & 2.259 .417 \\
\hline & 2008 & $56,3 \%$ & $28,2 \%$ & $15,5 \%$ & $100 \%$ & 2.392 .265 \\
\hline \multirow[t]{3}{*}{8} & 1998 & $37,4 \%$ & $46,3 \%$ & $16,3 \%$ & $100 \%$ & 1.892 .376 \\
\hline & 2003 & $43,6 \%$ & $41,5 \%$ & $14,9 \%$ & $100 \%$ & 2.397 .149 \\
\hline & 2008 & $41,5 \%$ & $40,7 \%$ & $17,9 \%$ & $100 \%$ & 2.642 .542 \\
\hline \multirow[t]{3}{*}{9} & 1998 & $22,1 \%$ & $58,0 \%$ & $19,9 \%$ & $100 \%$ & 1.995 .373 \\
\hline & 2003 & $26,0 \%$ & $57,8 \%$ & $16,2 \%$ & $100 \%$ & 2.649 .057 \\
\hline & 2008 & $25,0 \%$ & $53,6 \%$ & $21,4 \%$ & $100 \%$ & 2.819 .122 \\
\hline \multirow[t]{3}{*}{10} & 1998 & $12,8 \%$ & $64,0 \%$ & $23,2 \%$ & $100 \%$ & 2.293 .728 \\
\hline & 2003 & $14,9 \%$ & $68,1 \%$ & $17,0 \%$ & $100 \%$ & 2.761 .387 \\
\hline & 2008 & $16,8 \%$ & $61,1 \%$ & $22,1 \%$ & $100 \%$ & 3.066 .104 \\
\hline
\end{tabular}

*CV de 0,25 a 0,5. ${ }^{* *}$ CV acima de 0,5. Fonte: PNAD (1998, 2003 e 2008). No total foram incluídos outros atendimentos: Consulta com Agentes Comunitários de Saúde ou parteira; Consulta a farmácia; vacinação,injeção, curativos e pronto atendimentos; e, gesso ou imobilização.

O gasto privado direto financiou apenas 14\% dos atendimentos em nível nacional. Sua participação continua sendo, em 2008, concentrada nas consultas odontológicas $(51,6 \%)$. Porém a participação do SUS aumentou, financiando em 2008 pouco mais de $30 \%$, certamente como consequência do Programa de Saúde da Família ter incorporado esse profissional nas equipes ${ }^{5,6}$ e da implantação do Programa Brasil Sorridente em 2004. Por outro lado, apesar da expansão mais acentuada dos planos odontológicos do que os demais tipos de planos na última década ${ }^{6}$, sua participação no financiamento das consultas odontológicas diminuiu, dando cobertura em 2008 a apenas $17,9 \%$ das mesmas. É importante mencionar, que o número de pessoas que decla- raram ter usado consultas odontológicas no Brasil cresceu significativamente (90\%) em 2008 em comparação com 2003, o que, como apontado por Viacava ${ }^{4}$, confirma o aumento do acesso a consultas odontológicas. Em especial o SUS mais que duplicou, entre 2003 e 2008, o número de consultas por ele financiadas.

Em relação à evolução verificada no período 1998-2008 os maiores aumentos da participação do SUS se deu no financiamento das consultas médicas (de $58,6 \%$ para $62,7 \%$ ) e na realização de exames complementares (de $51,5 \%$ para $59,2 \%$ ). Entretanto, esse aumento foi registrado entre os anos 1998 e 2003, sendo as percentagens verificadas em 2008 similares ou inferiores às de 2003. O aumento da participação dos planos privados se 
deu exclusivamente no financiamento dos casos tratados por quimio e radioterapia, hemodiálise e hemoterapia (de 19,4\% em 2003 para 24,2\% em 2008). Assim, apesar de sua baixa participação no financiamento desse tipo de atendimentos verificou-se uma tendência de aumento. Por sua vez, o aumento da participação do gasto privado direto foi no financiamento de cirurgias ambulatoriais (de $7,8 \%$ para $11,7 \%$ ) e na realização de exames complementares $(8,9 \%$ para $10,6 \%)$.

\section{Atendimentos por região,} segundo sistema de financiamento

A participação do SUS no financiamento dos atendimentos é predominante em todas as regiões do país, especialmente no Norte e Nordeste (onde ela cobre $71 \%$ dos mesmos). Ela se elevou consideravelmente em todas as regiões entre 1998 e 2003 (Tabela 1). Este fato pode estar relacionado à expansão do Programa de Saúde da Família, que se deu de forma mais acelerada no Nordeste e mais tardia no Norte e Sudeste ${ }^{7}$.

Em 2008, se observa uma queda na participação relativa do SUS, especialmente nas regiões Norte, Nordeste e Sudeste. Esse decréscimo do protagonismo do SUS entre 2003 e 2008 foi compensado pelo aumento da participação do gasto privado direto, em todas as regiões, mantendo-se a dos planos de saúde constante nesse período.

\section{Atendimentos por decil de renda da população, segundo sistema de financiamento}

Ao analisar o financiamento dos atendimentos utilizados em 2008 pela população ordenada por renda, verificou-se que o SUS é predominante na utilização desses serviços até o sétimo decil. Ela cobre $88 \%$ dos atendimentos realizados pelo decil mais pobre e $56 \%$ dos utilizados pelo sétimo decil. Vale mencionar que o SUS financiou $17 \%$ dos atendimentos usados pelo decil mais rico (Tabela 1).

Observa-se um aumento da participação do SUS (de 13\% a 17\%) no financiamento dos atendimentos realizados para esse decil mais rico, provavelmente devido aos mecanismos de controle da utilização de serviços praticados pelas operadoras de planos de saúde descritos em Ugá et al. ${ }^{8}$ Nesse decil, o gasto privado direto não é de pouca importância, responsabilizando-se por $22 \%$ desses atendimentos em 2008 e sua participação se elevou significativamente entre 2003 e 2008 (passando de $17 \%$ a $22 \%$ ).

No que diz respeito aos dois decís mais pobres, a participação do SUS se manteve em 2008 praticamente a mesma que em 1998. Entretanto, decresceu quando comparada a 2003: ela caiu de $93 \%$ a $88 \%$ no primeiro decil e de $90 \%$ a $86 \%$ no segundo décimo da população. Esse fenômeno se verifica até o nono decil, embora apresente taxas de variação menos acentuadas; em contraposição, cresceu significativamente, nesses decís, a participação do gasto direto do bolso entre 2003 e 2008, mantendo-se a participação dos planos de saúde aproximadamente a mesma nesse período.

\section{Internações}

\section{Internações}

\section{segundo sistema de financiamento}

Em 2008, da mesma forma que nos anos anteriores, o SUS foi o responsável pelo financiamento da maior parte das internações efetuadas no país, 69,6\% das mesmas, segundo a PNAD, foram financiadas pelo setor público. A principal atuação do SUS no financiamento de internações se deu em partos normais $(91,7 \%)$, tratamento psiquiátrico $(78,1 \%)$ e tratamento clínico $(76,3 \%)$. Sua participação foi inferior, mas também importante, no financiamento de internações para a realização de exames $(68,9 \%)$ e para cirurgias (52,7\%) (Tabela 2).

No que concerne ao financiamento privado, no caso das internações, predominou nesse período a participação do segmento de planos de saúde, responsável por $24,8 \%$ das internações realizadas no país e, dentre estas, financiando $38,9 \%$ das internações cirúrgicas e $31 \%$ das referentes a partos cesáreos. O gasto privado direto cobriu apenas $5,7 \%$ das internações (principalmente as inerentes a cirurgias e parto cesáreo), provavelmente devido ao fato de que as internações, em geral, são de um custo elevado e podem frequentemente representar uma percentagem elevada da renda familiar, constituindo-se, assim, muitas vezes, em um gasto catastrófico que as pessoas tentam evitar comprando planos de saúde privados e/ou utilizando os serviços do SUS.

Considerando a evolução da participação de cada sistema de financiamento das internações efetuadas no país no período de 1998-2008, observa-se que cresceu o protagonismo do SUS (de $67,1 \%$ a $69,6 \%$ ), mas esse aumento ocorreu em 2003, mantendo-se a participação estatal relativamente constante em 2008. Em contrapartida, na década estudada decresceu a participação dos planos de saúde (de $25,5 \%$ para $24,8 \%$ ) e o gasto privado direto (de $7,3 \%$ para $5,7 \%$ ) no percentual de internações financiadas. 
Tabela 2. Internações por tipo, região e decil de renda, segundo financiamento - Brasil, 1998-2003-2008.

\begin{tabular}{|c|c|c|c|c|c|c|}
\hline & Ano & SUS & $\begin{array}{c}\text { Plano } \\
\text { Privado }\end{array}$ & $\begin{array}{c}\text { Gasto Privado } \\
\text { Direto }\end{array}$ & Total & $\mathbf{N}$ \\
\hline \multicolumn{7}{|l|}{ Motivo da internação } \\
\hline \multirow[t]{3}{*}{ Tratamento clínico } & 1998 & $72,3 \%$ & $21,5 \%$ & $6,2 \%$ & $100 \%$ & 5.459 .258 \\
\hline & 2003 & $75,8 \%$ & $20,0 \%$ & $4,2 \%$ & $100 \%$ & 6.394 .136 \\
\hline & 2008 & $76,3 \%$ & $19,5 \%$ & $4,1 \%$ & $100 \%$ & 7.151 .090 \\
\hline \multirow[t]{3}{*}{ Parto normal } & 1998 & $87,0 \%$ & $10,1 \%$ & $2,9 \%$ & $100 \%$ & 1.196 .781 \\
\hline & 2003 & $90,5 \%$ & $7,9 \%$ & $1,6 \%$ & $100 \%$ & 1.094 .227 \\
\hline & 2008 & $91,7 \%$ & $6,5 \%$ & $1,8 \%$ & $100 \%$ & 868.498 \\
\hline \multirow[t]{3}{*}{ Parto cesáreo } & 1998 & $55,5 \%$ & $32,0 \%$ & $12,5 \%$ & $100 \%$ & 896.288 \\
\hline & 2003 & $56,6 \%$ & $32,2 \%$ & $11,3 \%$ & $100 \%$ & 850.425 \\
\hline & 2008 & $58,4 \%$ & $31,1 \%$ & $10,5 \%$ & $100 \%$ & 987.293 \\
\hline \multirow[t]{3}{*}{ Cirurgia } & 1998 & $48,2 \%$ & $41,6 \%$ & $10,2 \%$ & $100 \%$ & 2.232 .913 \\
\hline & 2003 & $53,8 \%$ & $38,9 \%$ & $7,3 \%$ & $100 \%$ & 2.996 .027 \\
\hline & 2008 & $52,7 \%$ & $38,9 \%$ & $8,4 \%$ & $100 \%$ & 3.425 .286 \\
\hline \multirow[t]{3}{*}{ Tratamento psiquiátrico } & 1998 & $80,6 \%$ & $11,9 \%$ & $7,6 \% *$ & $100 \%$ & 164.399 \\
\hline & 2003 & $79,7 \%$ & $14,8 \%$ & $5,5 \% *$ & $100 \%$ & 181.049 \\
\hline & 2008 & $78,1 \%$ & $15,4 \%$ & $6,5 \%$ & $100 \%$ & 219.345 \\
\hline \multirow[t]{3}{*}{ Realização de exames } & 1998 & $58,7 \%$ & $31,3 \%$ & $9,9 \%$ & $100 \%$ & 239.350 \\
\hline & 2003 & $65,7 \%$ & $30,3 \%$ & $4,1 \%$ & $100 \%$ & 331.576 \\
\hline & 2008 & $68,9 \%$ & $26,1 \%$ & $5,0 \%$ & $100 \%$ & 487.149 \\
\hline \multirow[t]{3}{*}{ Total } & 1998 & $67,1 \%$ & $25,6 \%$ & $7,3 \%$ & $100 \%$ & 10.190 .593 \\
\hline & 2003 & $70,0 \%$ & $24,8 \%$ & $5,3 \%$ & $100 \%$ & 11.847 .440 \\
\hline & 2008 & $69,6 \%$ & $24,8 \%$ & $5,7 \%$ & $100 \%$ & 13.138 .661 \\
\hline \multicolumn{7}{|l|}{ Região } \\
\hline \multirow[t]{3}{*}{ Norte } & 1998 & $73,4 \%$ & $17,3 \%$ & $9,3 \%$ & $100 \%$ & 531.915 \\
\hline & 2003 & $79,1 \%$ & $14,7 \%$ & $6,2 \%$ & $100 \%$ & 780.798 \\
\hline & 2008 & $81,8 \%$ & $12,7 \%$ & $5,5 \%$ & $100 \%$ & 1.117 .560 \\
\hline \multirow[t]{3}{*}{ Nordeste } & 1998 & $83,1 \%$ & $13,7 \%$ & $3,2 \%$ & $100 \%$ & 2.840 .453 \\
\hline & 2003 & $84,5 \%$ & $12,5 \%$ & $3,0 \%$ & $100 \%$ & 3.317 .423 \\
\hline & 2008 & $83,9 \%$ & $12,5 \%$ & $3,7 \%$ & $100 \%$ & 3.587 .133 \\
\hline \multirow[t]{3}{*}{ Sudeste } & 1998 & $58,2 \%$ & $36,2 \%$ & $5,6 \%$ & $100 \%$ & 4.039 .244 \\
\hline & 2003 & $60,6 \%$ & $35,2 \%$ & $4,2 \%$ & $100 \%$ & 4.692 .890 \\
\hline & 2008 & $59,5 \%$ & $35,7 \%$ & $4,8 \%$ & $100 \%$ & 5.200 .444 \\
\hline \multirow[t]{3}{*}{ Sul } & 1998 & $63,3 \%$ & $23,9 \%$ & $12,8 \%$ & $100 \%$ & 1.942 .204 \\
\hline & 2003 & $65,1 \%$ & $25,6 \%$ & $9,3 \%$ & $100 \%$ & 2.018 .110 \\
\hline & 2008 & $65,4 \%$ & $25,7 \%$ & $8,9 \%$ & $100 \%$ & 2.056 .499 \\
\hline \multirow[t]{3}{*}{ Centro Oeste } & 1998 & $60,5 \%$ & $23,8 \%$ & $15,7 \%$ & $100 \%$ & 836.777 \\
\hline & 2003 & $68,1 \%$ & $22,5 \%$ & $9,4 \%$ & $100 \%$ & 1.038 .219 \\
\hline & 2008 & $66,2 \%$ & $23,6 \%$ & $10,2 \%$ & $100 \%$ & 1.177 .025 \\
\hline \multirow[t]{3}{*}{ Total } & 1998 & $67,1 \%$ & $25,6 \%$ & $7,3 \%$ & $100 \%$ & 10.190 .593 \\
\hline & 2003 & $70,0 \%$ & $24,8 \%$ & $5,3 \%$ & $100 \%$ & 11.847 .440 \\
\hline & 2008 & $69,6 \%$ & $24,8 \%$ & $5,7 \%$ & $100 \%$ & 13.138 .661 \\
\hline
\end{tabular}

Internações por região,

\section{segundo sistema de financiamento}

Da mesma forma que nos anos anteriores, em 2008, o SUS foi predominante no financiamento das internações em todas as regiões do país, principalmente, no Nordeste $(83,9 \%)$ e Norte $(81,8 \%)$. A participação dos planos se deu principalmente na região Sudeste (financiando
35,7\% das internações desse mesmo ano), dada a concentração da clientela do segmento de saúde suplementar nessa região (ANS, 2010), mas apresentou também percentuais importantes nas regiões Sul $(25,7 \%)$ e Centro- Oeste $(23,6 \%)$. A participação do gasto privado direto foi, como esperado, residual em todas as regiões, com as porcentagens mais elevadas nas regiões Sul e Cen- 
Tabela 2. continuação

\begin{tabular}{|c|c|c|c|c|c|c|}
\hline & Ano & SUS & $\begin{array}{c}\text { Plano } \\
\text { Privado }\end{array}$ & $\begin{array}{c}\text { Gasto Privado } \\
\text { Direto } \\
\end{array}$ & Total & $\mathbf{N}$ \\
\hline \multicolumn{7}{|c|}{ Decil de renda } \\
\hline \multirow[t]{3}{*}{1} & 1998 & $92,1 \%$ & $5,0 \%$ & $3,0 \%$ & $100 \%$ & 1.692 .975 \\
\hline & 2003 & $94,3 \%$ & $3,7 \%$ & $2,0 \%$ & $100 \%$ & 1.440 .400 \\
\hline & 2008 & $92,5 \%$ & $4,5 \%$ & $3,0 \%$ & $100 \%$ & 2.151 .960 \\
\hline \multirow[t]{3}{*}{2} & 1998 & $93,1 \%$ & $3,2 \%$ & $3,7 \%$ & $100 \%$ & 880.489 \\
\hline & 2003 & $92,9 \%$ & $4,8 \%$ & $2,3 \%$ & $100 \%$ & 1.538 .780 \\
\hline & 2008 & $91,5 \%$ & $5,8 \%$ & $2,7 \%$ & $100 \%$ & 902.829 \\
\hline \multirow[t]{3}{*}{3} & 1998 & $88,0 \%$ & $6,5 \%$ & $5,5 \%$ & $100 \%$ & 1.172 .818 \\
\hline & 2003 & $91,3 \%$ & $6,1 \%$ & $2,6 \%$ & $100 \%$ & 1.068 .467 \\
\hline & 2008 & $88,2 \%$ & $8,6 \%$ & $3,2 \%$ & $100 \%$ & 1.282 .719 \\
\hline \multirow[t]{3}{*}{4} & 1998 & $83,3 \%$ & $10,5 \%$ & $6,1 \%$ & $100 \%$ & 992.850 \\
\hline & 2003 & $86,4 \%$ & $9,0 \%$ & $4,6 \%$ & $100 \%$ & 1.254 .530 \\
\hline & 2008 & $84,7 \%$ & $10,0 \%$ & $5,3 \%$ & $100 \%$ & 1.434 .248 \\
\hline \multirow[t]{3}{*}{5} & 1998 & $74,4 \%$ & $17,7 \%$ & $7,9 \%$ & $100 \%$ & 1.100 .636 \\
\hline & 2003 & $79,6 \%$ & $16,0 \%$ & $4,5 \%$ & $100 \%$ & 1.219 .772 \\
\hline & 2008 & $77,3 \%$ & $17,3 \%$ & $5,4 \%$ & $100 \%$ & 1.259 .995 \\
\hline \multirow[t]{3}{*}{6} & 1998 & $65,1 \%$ & $25,9 \%$ & $9,0 \%$ & $100 \%$ & 855.904 \\
\hline & 2003 & $73,6 \%$ & $20,6 \%$ & $5,8 \%$ & $100 \%$ & 963.763 \\
\hline & 2008 & $74,3 \%$ & $19,5 \%$ & $6,1 \%$ & $100 \%$ & 1.281 .806 \\
\hline \multirow[t]{3}{*}{7} & 1998 & $55,3 \%$ & $36,3 \%$ & $8,4 \%$ & $100 \%$ & 844.302 \\
\hline & 2003 & $63,6 \%$ & $29,7 \%$ & $6,7 \%$ & $100 \%$ & 1.085 .339 \\
\hline & 2008 & $64,9 \%$ & $28,4 \%$ & $6,6 \%$ & $100 \%$ & 1.167 .633 \\
\hline \multirow[t]{3}{*}{8} & 1998 & $40,1 \%$ & $50,4 \%$ & $9,5 \%$ & $100 \%$ & 896.448 \\
\hline & 2003 & $49,4 \%$ & $42,7 \%$ & $7,9 \%$ & $100 \%$ & 1.036 .771 \\
\hline & 2008 & $50,8 \%$ & $41,7 \%$ & $7,5 \%$ & $100 \%$ & 1.180 .607 \\
\hline \multirow[t]{3}{*}{9} & 1998 & $25,7 \%$ & $61,2 \%$ & $13,2 \%$ & $100 \%$ & 844.329 \\
\hline & 2003 & $30,1 \%$ & $60,3 \%$ & $9,6 \%$ & $100 \%$ & 1.111 .755 \\
\hline & 2008 & $32,1 \%$ & $58,7 \%$ & $9,2 \%$ & $100 \%$ & 1.211 .505 \\
\hline \multirow[t]{3}{*}{10} & 1998 & $19,8 \%$ & $68,2 \%$ & $12,0 \%$ & $100 \%$ & 909.842 \\
\hline & 2003 & $19,0 \%$ & $71,6 \%$ & $9,4 \%$ & $100 \%$ & 1.112 .170 \\
\hline & 2008 & $23,4 \%$ & $67,8 \%$ & $8,8 \%$ & $100 \%$ & 1.242 .787 \\
\hline
\end{tabular}

* CV de 0,25 a 0,5. ${ }^{* *} \mathrm{CV}$ acima de 0,5. Fonte: $\operatorname{PNAD}(1998,2003$ e 2008).

tro-Oeste, $8,9 \%$ e $10,2 \%$ respectivamente para 2008 (Tabela 2).

Considerando a evolução da participação do SUS no financiamento das internações efetuadas no país no período de 1998-2008, observa-se que ela cresceu fundamentalmente na Região Norte (passando de $73,4 \%$ a $81,8 \%$ ), e no Centro-Oeste (passando de $60,5 \%$ a $66,2 \%$ ). Os planos privados apresentaram pequenas oscilações, excetuando na região Norte na qual sua participação no financiamento das internações diminuiu passando de 17,3\% em 1998 para 12,7\% em 2008. Em relação ao gasto privado direto observou-se uma diminuição de sua participação praticamente em todas as regiões (só na região Nordeste se manteve com a mesma participação), a diminui- ção se deu entre os anos de 1998 e 2003, mantendo em 2008 um participação similar à verificada para 2003.

\section{Internações por decil de renda da população, segundo sistema} de financiamento

Como esperado, o SUS continua predominando no financiamento das internações dos decís de mais baixa renda (até o sétimo decil); no oitavo ele cobre $50,8 \%$ das internações e, a partir do nono decil ele deixa de ser preponderante. Nos dois decís mais ricos, as internações são majoritariamente financiadas pelos planos privados de saúde (que cobrem 58,7\% das internações do nono decil de renda e $67,8 \%$ do décimo mais rico). 
Entretanto, é notório o aumento da participação do SUS no financiamento das internações realizadas pela população dos dois decís de maior renda, entre 2003 e 2008, fenômeno esse que provavelmente reflete o aumento da utilização de serviços SUS por pessoas portadoras de planos e seguros privados em decorrência dos mecanismos de controle do uso praticados nos sistemas de atenção gerenciada (Tabela 2).

Ao mesmo tempo verificou-se, entre 2003 e 2008, um crescimento, ainda que pequeno, da participação dos planos de saúde no financiamento das internações de pessoas situadas nos decís mais pobres (principalmente nos três primeiros décimos), provavelmente explicado pelo aumento da formalidade do emprego observado nos últimos anos (que passou de 57,6\% a $60,6 \%$ entre 2003 e $2007^{9}$ e pelo fato de que a maior parte dos planos de saúde são coletivos, isto é, promovidos ou organizados pelas empresas, segundo a ANS $^{6}$.

Ainda, observa-se, em valores absolutos, um aumento muito mais acentuado do número de internações efetuadas pela população do primeiro decil de renda do que nos demais, o que denota certamente uma ampliação do acesso a internações dessa população. Como se observa na Tabela 2, acima apresentada, esse crescimento se dá fundamentalmente em relação a internações para tratamento clínico e, secundariamente, para tratamento cirúrgico.

Vale mencionar, ainda, que o gasto privado direto não alcança, em 2008, 10\% em nenhum decil, e que, tendo em vista as observações acima apontadas, sobre a catastroficidade do gasto em boa parte das internações, sua participação no financiamento diminuiu em todos os decis.

\section{Internações SUS por decil de renda, segundo tipo de internação}

$\mathrm{O}$ tipo de internação predominantemente financiada pelo SUS, no ano de 2008, foi aquela destinada ao tratamento clínico, representando entre $58 \%$ a $62 \%$ nos distintos decís da população ordenada pela renda (Tabela 3).

Deve-se destacar que, enquanto das internações SUS destinadas ao primeiro decil de renda apenas $12,9 \%$ o foram para tratamento cirúrgico, das destinadas ao nono décimo populacional as cirúrgicas foram $39,8 \%$ e que a percentagem correspondente ao último decil foi $25,7 \%$. Isto sugere um maior acesso desses decis mais ricos a tratamentos cirúrgicos efetuados em regime de internação

\section{Desigualdades na utilização de serviços de saúde, segundo a posse ou não de plano de saúde}

Foram observadas importantes desigualdades nas taxas de utilização de atendimentos: 7,9\% dos usuários do SUS utilizaram atendimentos nos últimos quinze dias prévios à entrevista da PNAD2008 , enquanto $18,9 \%$ das pessoas que possuem plano de saúde os utilizaram (Tabela 4)

O mesmo ocorreu no caso das taxas de utilização de internações e entre as pessoas que usaram o SUS e aquelas que usaram serviços financiados pelo plano de saúde: enquanto $4,8 \%$ da população que usa o SUS se internou em 2008, $8 \%$ das pessoas com plano o fizeram (Tabela 4 ).

Tabela 3. Internações SUS por decil de renda, segundo tipo de internternação - 2008

\begin{tabular}{lcrrrcrrr}
\hline Decil & $\begin{array}{c}\text { Inter. p/ } \\
\text { tratamento } \\
\text { clínico }\end{array}$ & $\begin{array}{c}\text { Parto } \\
\text { normal }\end{array}$ & $\begin{array}{c}\text { Parto } \\
\text { Cesário }\end{array}$ & $\begin{array}{c}\text { Intern. p/ } \\
\text { cirurgia }\end{array}$ & $\begin{array}{c}\text { Intern. p/ } \\
\text { tratam. } \\
\text { Psiquiátrico }\end{array}$ & $\begin{array}{c}\text { Intern. p/ } \\
\text { exames }\end{array}$ & \% Total & N \\
\hline 1 & $58,7 \%$ & $14,2 \%$ & $8,5 \%$ & $12,9 \%$ & $2,0 \%$ & $3,8 \%$ & $100 \%$ & 1.989 .982 \\
2 & $60,4 \%$ & $10,0 \%$ & $8,4 \%$ & $16,9 \%$ & $1,5 \% *$ & $2,8 \%$ & $100 \%$ & 826.520 \\
3 & $58,9 \%$ & $10,7 \%$ & $7,8 \%$ & $18,1 \%$ & $1,2 \%$ & $3,2 \%$ & $100 \%$ & 1.131 .856 \\
4 & $62,3 \%$ & $6,5 \%$ & $5,4 \%$ & $20,6 \%$ & $2,3 \%$ & $2,8 \%$ & $100 \%$ & 1.214 .824 \\
5 & $60,4 \%$ & $8,2 \%$ & $5,4 \%$ & $20,6 \%$ & $1,5 \%$ & $3,9 \%$ & $100 \%$ & 973.814 \\
6 & $60,1 \%$ & $5,5 \%$ & $4,8 \%$ & $21,7 \%$ & $3,4 \%$ & $4,5 \%$ & $100 \%$ & 952.922 \\
7 & $58,8 \%$ & $5,3 \%$ & $5,0 \%$ & $25,3 \%$ & $1,8 \% *$ & $3,9 \%$ & $100 \%$ & 758.371 \\
8 & $59,1 \%$ & $5,5 \%$ & $3,4 \%$ & $26,7 \%$ & $1,2 \% *$ & $4,1 \%$ & $100 \%$ & 599.718 \\
9 & $58,4 \%$ & $2,5 \% *$ & $3,8 \%$ & $29,8 \%$ & $1,4 \% * *$ & $4,2 \%$ & $100 \%$ & 389.024 \\
10 & $57,8 \%$ & $5,6 \%$ & $4,6 \% *$ & $25,7 \%$ & $1,3 \% * *$ & $4,9 \%$ & $100 \%$ & 290.212 \\
\hline
\end{tabular}

${ }^{*} \mathrm{CV}$ de 0,25 a 0,5. ${ }^{* *} \mathrm{CV}$ acima de 0,5. Fonte: PNAD (1998, 2003 e 2008) 
Tabela 4. Proporção de pessoas atendidas e internadas, segundo seu financiamento. Brasil, 1998- 2003-2008.

\begin{tabular}{|c|c|c|c|c|c|c|c|c|c|}
\hline & \multicolumn{3}{|c|}{ Número } & \multicolumn{3}{|c|}{$\begin{array}{l}\text { Proporção de } \\
\text { atendimentos em } \\
\text { relação ao total } \\
\text { população brasileira }\end{array}$} & \multicolumn{3}{|c|}{$\begin{array}{c}\text { Proporção de } \\
\text { atendimentos em } \\
\text { relação à população } \\
\text { brasileira com plano }\end{array}$} \\
\hline & 1998 & 2003 & 2008 & 1998 & 2003 & 2008 & 1998 & 2003 & 2008 \\
\hline \multicolumn{10}{|l|}{ Atendimento } \\
\hline $\begin{array}{l}\text { Total de pessoas atendidas } \\
\text { no país }\end{array}$ & 20.129 .225 & 25.143 .708 & 26.866 .869 & 12,7 & 14,3 & 14,1 & - & - & - \\
\hline $\begin{array}{l}\text { Pessoas que foram atendidas } \\
\text { financiadas pelo SUS }\end{array}$ & 9.825 .712 & 14.270 .984 & 14.952 .129 & 6,2 & 8,1 & 7,9 & - & - & - \\
\hline $\begin{array}{l}\text { Pessoas que foram atendidas } \\
\text { financiadas pelo plano }\end{array}$ & 7.190 .871 & 8.517 .408 & 9.318 .225 & - & - & - & 18,6 & 19,8 & 18,9 \\
\hline $\begin{array}{l}\text { Pessoas com plano que foram } \\
\text { atendidas financiadas pelo SUS }\end{array}$ & 652.496 & 1.004 .759 & 1.216 .499 & - & - & - & 1,7 & 2,3 & 2,5 \\
\hline \multicolumn{10}{|l|}{ Internações } \\
\hline $\begin{array}{l}\text { Total de pessoas internadas } \\
\text { no país }\end{array}$ & 10.981 .824 & 12.332 .901 & 13.513 .509 & 6,9 & 7,0 & 7,1 & - & - & - \\
\hline $\begin{array}{l}\text { Pessoas internadas financiadas } \\
\text { pelo SUS }\end{array}$ & 6.838 .218 & 8.287 .552 & 9.142 .712 & 4,3 & 4,7 & 4,8 & - & - & - \\
\hline $\begin{array}{l}\text { Pessoas internadas financiadas } \\
\text { pelo plano }\end{array}$ & 3.110 .581 & 3.572 .886 & 4.018 .489 & - & - & - & 8,0 & 8,3 & 8,2 \\
\hline $\begin{array}{l}\text { Pessoas com plano de saúde } \\
\text { internadas financiadas pelo SUS }\end{array}$ & 389.158 & 553.036 & 670.400 & - & - & - & 1,0 & 1,3 & 1,4 \\
\hline
\end{tabular}

Fonte: PNAD (1998, 2003 e 2008)

Em que pesem essas diferenças na utilização de serviços entre usuários do SUS e de planos de saúde, observa-se no decênio 1998-2008 um contínuo aumento da taxa de utilização do SUS: ela passou de $6,2 \%$ a $7,9 \%$ no que concerne aos atendimentos e de $4,3 \%$ a $4,8 \%$ no que diz respeito às internações. Este aumento mais acentuado no uso de atendimentos SUS do que no das internações está provavelmente relacionado à expansão de cobertura do Programa de Saúde da Família ${ }^{10}$ no período estudado. Atualmente, segundo a PNAD, cerca de $50 \%$ dos domicílios estão cadastrados nesse Programa, encontrando-se a maior taxa de cobertura (quase 70\%) na região Nordeste e a menor (39\%) na Sudeste ${ }^{4}$.

Diferentemente, as taxas de utilização das pessoas que possuem planos de saúde privados mantiveram-se praticamente constantes, passando de $18,6 \%$ a $18,9 \%$ no caso dos atendimentos e de $8,0 \%$ a $8,2 \%$ no caso das internações. Como já demonstrado em alguns estudos ${ }^{8}$, as operadoras de planos de saúde exercem fortes mecanismos de controle da utilização de serviços de saúde, que se expressam na estabilidade das taxas acima mencionadas.

Em correspondência a esse movimento, cresceu a porcentagem dos serviços SUS usados por pessoas que possuem plano de saúde, entre 1998 e 2008, entretanto o maior aumento se deu no período 1998-2003, sendo as percentagens observadas em 2008 similares às verificadas em 2003.

Esse percentual cresceu significativamente (Tabela 5), principalmente no caso de cirurgias em ambulatórios, nos exames complementares e em outros tipos de atendimentos que incluem vacinações, injeções, gessos etc. Cabe destacar que o comportamento dos outros tipos de atendimento está certamente influenciado pela vacinação, atividade de prevenção desenvolvida pelos serviços públicos de saúde.

Como já apontado em Santos et al. ${ }^{11}$, os atendimentos SUS mais usados por pessoas possuidoras de plano de saúde são, por um lado, os de baixa complexidade - vacinação, injeção e, de outro, os de alta complexidade e alto custo quimioterapia, radioterapia, hemodiálise, hemoterapia sobre os quais recai um forte controle das operadoras de planos de saúde no sentido da contenção de sua utilização.

Também se observa um importante crescimento do percentual de todos os tipos de internações SUS usadas por clientes de planos de saúde, principalmente no que se refere a partos normais (de $5 \%$ a $7 \%$ ) e cesáreos (de $6 \%$ a $7 \%$ ) e às 
Tabela 5. Proporção de atendimento e internações financiadas pelo SUS, segundo tipo de serviço, por parte da população com plano privado de saúde. Brasil, 1998 - 2003-2008.

\begin{tabular}{lrrr}
\hline & \multicolumn{3}{c}{$\%$} \\
\cline { 2 - 4 } & $\mathbf{1 9 9 8}$ & $\mathbf{2 0 0 3}$ & $\mathbf{2 0 0 8}$ \\
\hline Tipo de atendimento & & & \\
$\quad$ Consulta médica & 6,2 & 6,5 & 7,5 \\
$\quad$ Consulta odontológica & 7,0 & 7,4 & 8,2 \\
Consulta de outro profissional & $7,4^{*}$ & 8,7 & 9,8 \\
Quimioterapia, radioterapia, hemodiálise, hemoterapia & $\mathrm{n} / \mathrm{a}$ & $11,5^{*}$ & $12,8^{*}$ \\
Cirurgia em ambulatório & $5,8^{*}$ & 7,2 & 8,7 \\
Exames complementares & 6,4 & 8,4 & 8,8 \\
Outro atendimento & 9,3 & 9,5 & 13,8 \\
$\quad$ \% de pessoas com plano atendidas pelo SUS & 6,6 & 7,0 & 8,1 \\
Tipo de internação & & & 6,8 \\
Tratamento clínico & 5,2 & 6,0 & 7,4 \\
Parto normal & 4,8 & 6,0 & 7,2 \\
Parto cesáreo & 5,8 & 7,3 & 8,8 \\
Cirurgia & 8,4 & 9,0 & 8,1 \\
Tratamento psiquiátrico & $3,5^{*}$ & $4,2^{*}$ & 8,4 \\
Exames & 6,0 & 7,7 & 7,3 \\
$\quad \%$ de pessoas com plano internadas pelo SUS & 5,7 & 6,7 & \\
\hline
\end{tabular}

${ }^{*} \mathrm{CV}$ de 0,25 a 0,5. $\quad{ }^{* *} \mathrm{CV}$ acima de 0,5. Fonte: $\operatorname{PNAD}(1998,2003$ e 2008).

internações para realização de exames (de $6 \%$ a 8\%) (Tabela 5). Isto também remete em parte à urgência nos casos dos partos e aos mecanismos de controle da utilização de serviços praticados pelas operadoras de planos de saúde, acima mencionados, no caso das internações para realização de exames.

Vale destacar, também, que o maior percentual da utilização de internações SUS por clientes de planos privados se observa em relação às internações para cirurgias: nada menos que $9 \%$ das cirurgias realizadas pelo SUS foram usadas por essas pessoas que têm dupla cobertura na atenção à saúde.

Este fenômeno se reflete em um expressivo aumento das taxas de utilização de serviços SUS por clientes de planos de saúde, tanto no que se refere a atendimentos (cujo aumento foi de $47 \%$ ) como a internações (cuja taxa cresceu $40 \%$ ).

\section{Discussão}

O SUS continua sendo o principal financiador dos serviços utilizados pela população brasileira, nos atendimentos efetuados em ambulatórios e mais ainda no caso das internações. Entretanto é importante destacar que o expressivo crescimento do protagonismo do SUS observado entre 1998 e
2003 não se deu entre 2003 e 2008. Cabe destacar o aumento da participação do SUS no financiamento dos atendimentos odontológicos, que em 2008 foi pouco mais de $30 \%$, certamente é consequência do Programa de Saúde da Família ter incorporado esse profissional nas equipes e da implantação do Programa Brasil Sorridente. O SUS mais que duplicou, entre 2003 e 2008, o número de consultas por ele financiadas.

A participação do SUS no financiamento da utilização dos serviços de saúde foi preponderante em todas as regiões, sendo maior nas regiões Norte e Nordeste com situações sanitárias e sócio-econômicas mais precárias.

Sua participação se mantém concentrada nas populações de mais baixa renda, apresentando, em 2008, duas mudanças importantes: por um lado um aumentando significativo do número de atendimentos e internações financiados para a população do primeiro decil de renda, indicando uma melhoria no acesso dessa população, e por outro um crescimento de sua participação no financiamento dos atendimentos do último decil e das internações dos dois decis mais ricos. Esse aumento na participação relativa do uso de serviços das populações mais abastadas é coerente com o aumento do uso do SUS por parte da população que conta com planos e seguros privados de saúde. 
Outro resultado que merece ser destacado, e futuramente mais analisado, diz respeito ao indicativo de desigualdades no acesso às internações cirúrgicas financiadas pelo SUS. A diferença das porcentagens de internações cirúrgicas entre o primeiro e os últimos decis apontam para um favorecimento no acesso a esse tipo de internações SUS das pessoas com maior renda.

A participação dos planos privados de saúde diminuiu tanto em relação ao financiamento de atendimentos ambulatoriais quanto ao de internações. Essa diminuição se deu em todos os tipos de atendimentos, excetuando os relativos a quimio e radioterapia, hemodiálise e hemoterapia. Essa diminuição da participação no financiamento dos atendimentos se deu fundamentalmente nas regiões Norte, Nordeste e Sudeste, e no financiamento dos atendimentos correspondentes as populações dos cinco últimos decis de renda. No caso das internações a diminuição foi constatada para todos os tipos de internações, excetuando as motivadas por problemas psiquiátricos. As regiões mais afetadas por essa queda na participação dos planos privados no financiamento das internações foram Norte e Nordeste. $\mathrm{Na}$ analise por decis de renda destaca-se seu amento nos primeiros decis, como mencionado, provavelmente produto do aumento da formalidade no emprego, e a queda na participação do financiamento das internações efetuadas pelas populações dos quatro últimos decis.

O gasto privado direto, como é lógico, dados os custos correspondentes, tem uma maior participação no financiamento dos atendimentos ambulatoriais, com preponderância nas consultas odontológicas. Ele tem uma maior participação nos atendimentos das regiões Sul e CentroOeste, e sua participação aumenta na medida em que aumenta a renda da população. Em relação a sua participação no financiamento das internações é importante assinalar que vem diminuindo em todos os tipos de internação, em todas as regiões e no financiamento das internações das populações de todos os decis de renda.

Finalmente, destaca-se ainda que, apesar do aumento verificado nas taxas de utilização do SUS, para atendimentos e internações, ainda persistem grandes iniquidades no uso de serviços de saúde entre a população atendida pelo SUS e a população beneficiária de planos e seguros de saúde privados, mais ainda quando considerado o aumento observado em relação à utilização de serviços do SUS por parte dessa população com asseguramento privado.

\section{Conclusão}

O SUS continua sendo o principal financiador dos serviços utilizados pela população brasileira, nos atendimentos efetuados em ambulatórios e mais ainda no caso das internações. Entretanto é importante destacar que o expressivo crescimento do protagonismo do SUS observado entre 1998 e 2003 não se deu entre 2003 e 2008. Ainda assim, algumas melhorias dos últimos cinco anos merecem ser destacadas, por exemplo, o aumento do acesso aos serviços de saúde da população do primeiro decil de renda e a maior cobertura nos atendimentos odontológicos. Porém, é necessário reconhecer que entre 2003 e 2008 pouco se avançou no alcance dos princípios norteadores do SUS: universalidade, integralidade e equidade. As desigualdades entre as taxas de utilização do SUS e as estimadas para a população portadora de planos privados de saúde praticamente continuou inalterada.

Finalmente, vale destacar a importância da realização regular do suplemento referente à saúde da PNAD. Como mostra este artigo, graças a ela foi possível mensurar a evolução do SUS em termos de cobertura, bem como o comportamento do segmento de saúde suplementar e do gasto privado. 


\section{Colaboradores}

SMPorto e MAD Ugá participaram na concepção, análise e redação do artigo, e RS Moreira realizou o tratamento estatístico dos dados.

\section{Referências}

1. Silva PLN, Pessoa DGC, Lila MF. Análise estatística de dados da PNAD: incorporando a estrutura do plano amostral. Cien Saude Colet 2002; 7(4): 659-670.

2. Porto SM, Santos IS, Ugá MAD. A utilização de serviços de saúde por sistema de financiamento. Cien Saude Colet 2006; 11(4):895-910.

3. IBGE. Acesso e utilização de serviços de saúde PNAD 2003. Rio de Janeiro: Instituto Brasileiro de Geografia e Estatística; 2005.

4. Viacava F. Acesso e uso de serviços de saúde pelos brasileiros. Radis 2010; 96:12-19. [acessado 2010 ago]. Disponível em: www.ensp.fiocruz.br/radis

5. Brasil. Ministério da Saúde. Portaria n 1.444/GM Estabelece incentivo financeiro para a reorganização da atenção à saúde bucal prestada nos municípios por meio do Programa de Saúde da Família. Diário Oficial da República Federativa do Brasil 2000; 28 dez.

6. Brasil. Agência Nacional de Saúde Suplementar (ANS). Caderno de Informação da Saúde Suplementar: beneficiários, operadoras e planos. Rio de Janeiro: ANS; Junho de 2010. [acessado 2010 ago 20] Disponível em: http://www.ans.gov.br/portal/site/informacoesss /informacoesss.asp

7. Giovanella L, Mendonça MH. Atenção Primária à Saúde. In: Giovanella, L, Lobato L, Escorel S, Carvalho A, Noronha J, organizadores. Politicas e Sistema de Saúde no Brasil. Rio de Janeiro: Editora Fiocruz; 2008.

8. Ugá MAD, Vasconcellos MM, Lima SM, Portela MC, Gerschman S. Mecanismos de microrregulação aplicados por operadoras de planos de saúde sobre hospitais privados. Rev Saude Publica 2009; 43(5):832-838.

9. Instituto de Pesquisa Econômica Aplicada. (IPEADATA). [acessado 2010 ago 20]. Disponível em: http://www.ipeadata.gov.br/

10. Schilling C. Saúde da Família - desafios para a próxima década. Brasília: Departamento de Atenção Básica; 2010. [acessado 2011 jan]. Disponível em: http:/ /www.conass.org.br/arquivos/file/apresentacao_ claunara_schilling_saude_da_familia_bsb_26_julho 2010.pdf

11. Santos IS, Ugá MAD, Porto SM. O mix públicoprivado no Sistema de Saúde Brasileiro: financiamento, oferta e utilização de serviços de saúde. Cien Saude Colet 2008; 13(5):1431-1440.

Artigo apresentado em 01/02/2011

Aprovado em 01/04/2011

Versão final apresentada em 10/05/2011 